FORMATION Formation emploi

Revue française de sciences sociales

146 | Avril-Juin 2019

L'apprentissage en Allemagne face à ses défis

\title{
Les compétences sociales dans la formation par apprentissage en Allemagne : l'enseignement professionnel fondé sur les compétences
}

Social competences in German apprenticeship training within competence:

based vocational education

Soziale Kompetenzen in der beruflichen Ausbildung in Deutschland aus

kompetenzbasierter Perspektive

Las competencias sociales en la formación alemana en aprendizaje - la

enseñanza técnica basada en las competencias

Agnes Dietzen et Tanja Tschöpe

\section{OpenEdition}

Journals

Édition électronique

URL : http://journals.openedition.org/formationemploi/7377

DOI : 10.4000/formationemploi.7377

ISSN : 2107-0946

Éditeur

La Documentation française

Édition imprimée

Date de publication : 26 juin 2019

Pagination : 77-99

ISSN : 0759-6340

Référence électronique

Agnes Dietzen et Tanja Tschöpe, «Les compétences sociales dans la formation par apprentissage en Allemagne : I'enseignement professionnel fondé sur les compétences », Formation emploi [En ligne], 146 | Avril-Juin 2019, mis en ligne le 01 janvier 2021, consulté le 07 janvier 2021. URL : http:// journals.openedition.org/formationemploi/7377 ; DOI : https://doi.org/10.4000/formationemploi.7377 


\title{
Les compétences sociales dans la formation par apprentissage en Allemagne : l'enseignement professionnel fondé sur les compétences
}

\author{
Agnes Dietzen \\ Sociologue, directrice de recherches et directrice du département de recherche \\ Développement des compétences, au sein de l'Institut fédéral pour la formation et \\ l'enseignement professionnels (BIBB) \\ TANJA TSCHÖPE \\ Psychologue et chercheuse au sein du département Développement des compétences de \\ I'Institut fédéral pour la formation et l'enseignement professionnels (BIBB)
}

Résumé

Les compétences sociales dans la formation par apprentissage en Allemagne : l'enseignement professionnel fondé sur les compétences

Le présent article aborde les compétences sociales dans l'enseignement et la formation professionnels (EFP), ainsi que dans la formation initiale, puisqu'elles constituent une composante essentielle de la compétence professionnelle dans la plupart des secteurs professionnels. Adoptant une démarche de recherche empirique sur les compétences dans l'EFP, nous estimons qu'il est nécessaire de clarifier l'architecture des compétences sociales au regard des caractéristiques propres à chaque métier. L'article présente une manière d'élaborer et de valider un modèle s'agissant du métier d'assistant.e médical.e (en allemand, "medizinische/r Fachangestellte/r » est un métier qui se situe entre celui de secrétaire-médical.e et d'assistant.e). Nous mobilisons les résultats empiriques issus de l'évaluation des compétences sociales des assistant·e-s médicaux-ales, après trois années de formation initiale. Ainsi, nous préconisons un développement de la formation d'assistant.e médical.e incorporant davantage certaines dimensions des compétences sociales.

Mots clés : apprentissage, compétence relationnelle, enseignement techniqueprofessionnel, secrétaire, santé, Allemagne 
Abstract

Social competences in German apprenticeship training within competence: based vocational education

This paper addresses social competences in vocational education and training (VET) and initial training since they form basic components of professional competence in most occupational sectors. From an empirical competence research approach in VET, it argues that there is a need for a clarification of the construct of social competences regarding its occupation-specific features. We present how to develop and validate such a model for the case of medical assistants. Based on empirical results for the measuring of social competences of medical assistants after three years of initial training, we recommend a further development of training for medical assistants with regard to certain dimensions of social competences.

Keywords: apprenticeship, relationship skills, technical \& vocational education, secretary, healthcare, Germany

Journal of Economic Literature: I 29 ; J 24

Traduction : Auteures.

\section{Introduction}

Incontestablement, les compétences sociales sont essentielles pour répondre aux exigences professionnelles dans de nombreux champs d'activité, et il est communément admis que le besoin en compétences sociales va continuer de croître dans un monde du travail numérisé. Pour le système allemand d'enseignement et de formation professionnels, les compétences sociales sont indispensables à l'acquisition et à l'exercice de la compétence professionnelle.

Depuis les années 1990, le développement et la promotion des compétences sociales font partie des objectifs d'enseignement et de formation inscrits dans les programmes de formation en entreprise et en milieu scolaire. Toutefois, il apparaît que les examens actuels ne tiennent pas suffisamment compte de ces objectifs (cf. Lorig \& al, 2014). En outre, les approches existantes en faveur du développement des compétences sociales pendant la formation sont depuis longtemps perçues comme inadaptées et posent problème dans la plupart des cas (Seyfried, 1995 ; Euler, Bauer-Klebl, 2008 ; Euler, 2012 ; Tschöpe, Monnier, 2016).

Des efforts considérables doivent donc impérativement être entrepris afin de mieux promouvoir les compétences sociales dans la pratique de formation. En tant qu'objet de réflexion, les compétences sociales soulèvent de nombreuses questions, dont beaucoup 
restent encore sans réponse et dont certaines sont fondamentales. En effet, des recherches approfondies révèlent qu'il n'existe pas de définition claire du terme " compétences sociales ". De nombreux concepts et notions existent, mais ne consistent souvent qu'en une juxtaposition de différents termes relevant du concept sémantique de comportement social. Ils se fondent pour beaucoup sur une plausibilité supposée et ne sont pas suffisamment justifiés au plan théorique, ni prouvés par des données empiriques (Kanning, 2005). Quelles sont précisément ces compétences et quelle terminologie employer pour les délimiter et les distinguer les unes des autres ? Ces questions restent en suspens et un travail cohérent de recherche empirique et conceptuelle est nécessaire pour y répondre.

Une autre question non encore clarifiée est celle de la distinction entre les compétences sociales générales et celles propres à un métier. Faut-il aligner les compétences sociales sur des compétences transversales ou convient-il plutôt de les décliner au cas par cas en compétences-métiers ? Ce problème a été traité de façon précoce, puisque cela remonte au débat sur les qualifications transversales qui a eu lieu, en Allemagne, dans les années 1970. Face à la dynamique de changement des métiers et de l'emploi, Mertens (1974) a appelé à instaurer des qualifications-clés transversales. Il s'agissait de former des travailleurs plus flexibles, dotés d'une gamme de compétences de base plus ample pour forger leur employabilité. Pour ce faire, l'idée était que les profils de qualifications visés comportent également des "compétences sociales", dont "la capacité à communiquer et à coopérer, à résoudre des conflits par voie rationnelle, à passer d'un rôle social à un autre ainsi qu'un certain nombre d'aptitudes liées au travail et à la motivation" (Mertens 1974, p. 40 sq.). Le débat sur les qualifications transversales, qui a fait suite aux travaux de Mertens, répond clairement au souci de développer une main-d'œuvre flexible. Ces discussions ont abouti à plusieurs classements systématiques des compétences sociales, sans pour autant établir de distinction probante entre les compétences-métiers et les compétences transversales, faute de logique systémique et conceptuelle.

Les critiques objectent à l'approche par les compétences transversales que la définition du comportement socialement compétent peut varier du tout au tout selon les contextes et les situations. Ainsi, un comportement perçu comme une aptitude à s'affirmer dans un contexte économique pourra, dans un cadre pédagogique, être vu comme un manque de coopération. Le jugement porté sur un comportement social est toujours fonction des valeurs et des attitudes des personnes impliquées, ainsi que des attentes comportementales qui prévalent dans la pratique professionnelle. Si les compétences sociales ont généralement une connotation positive dans un contexte donné, elles peuvent être jugées tout autrement dans un cadre où les valeurs et les normes en vigueur sont différentes, voire incompatibles (Kanning, 2005). En outre, les situations de travail sont susceptibles de varier sous l'effet d'autres données circonstancielles. Ainsi, travailler avec des clients ne requiert certainement pas les mêmes compétences que travailler avec des collègues. Il apparaît donc essentiel de distinguer différents types de situations professionnelles pour 
former aux compétences sociales, les évaluer et les mesurer (Schuler, Barthelme, 1995 ; Euler, Bauer Klebl, 2008).

Le débat actuel sur la formation n'a pas encore élucidé le rapport entre les caractéristiques transversales des compétences sociales et celles propres à un métier (Baethge \& al., 2006 ; Baethge, Seeber, 2016). Toutefois, ces dernières années, des travaux de recherche en formation et enseignement professionnels, ciblés sur les compétences, ont entrepris de fournir des explications conceptuelles reposant sur des analyses empiriques. Ces travaux indiquent qu'il s'agit en majeure partie de compétences propres au contexte et qu'il convient de les aborder métier par métier et en tenant compte des circonstances.

Au vu de ces travaux de recherche empirique sur les compétences dans l'EFP, il convient de clarifier l'architecture des compétences sociales, et notamment de leurs caractéristiques contextuelles. L'exemple que nous présentons illustre comment développer un modèle, empiriquement valide, de compétences sociales propres à un métier. Ce modèle constitue une base solide utile pour concevoir des formations aux compétences sociales propres à chaque métier et des évaluations de ces compétences.

L'argumentation débute par une brève mise en perspective des contextes d'analyse des compétences sociales et des activités de recherches correspondantes afin d'éclairer notre démarche de recherche. Nous présentons ensuite des exemples d'activités de recherche menées pour développer et valider un modèle de compétences sociales pour la profession d'assistant.e médical.e. Il s'agir du deuxième métier en apprentissage le plus choisi par les jeunes femmes (cf. Institut fédéral pour la formation et l'enseignement professionnels, [BIBB], 2017a), et qui comporte de nombreux défis en matière d'interaction. Enfin, nous nous demandons en quoi notre démarche peut contribuer à l'élaboration d'un concept de compétences sociales empiriquement prouvé et comment étayer la formation en répondant aux exigences propres à chaque métier. 


\section{Encadré 1. Assistant·e médical·e, formation et métier}

En Allemagne, le programme d'enseignement professionnel délivrant le certificat d'assistant.e médical.e est un cursus de trois ans en alternance. C'est le sixième métier en apprentissage le plus apprécié du système allemand d'EFP en alternance, avec au total 15708 nouveaux contrats d'apprentissage et 39984 aprenti.e.s pour l'année 2017. La profession se caractérise par une forte représentation féminine, puisque 97,6 \% des aprenti.e.s sont des femmes (cf. Institut fédéral pour la formation et l'enseignement professionnels, [BIBB], 2017c).

La plupart des assistant-es médicaux-ales trouvent un emploi dans les cabinets de consultation de médecins généralistes ou spécialistes, à l'hôpital ou chez d'autres prestataires de soins médicaux. Elles/ils peuvent également travailler dans des instituts de recherche et des laboratoires, dans des services médicaux en entreprise ou auprès d'autres institutions ou organismes du service de santé publique (cf. BIBB, 2007, 14).

Les activités liées à ce métier se caractérisent généralement par un lien étroit avec les patient.es et les membres de leurs familles. Ils/elles assument en effet un rôle de guide et de soutien avant, pendant et après un traitement médical et effectuent certains examens et actes, soit directement, soit sous le contrôle d'un médecin. Outre les compétences sanitaires et commerciales, les compétences sociales sont une composante fondamentale du profil de ce métier. Le règlement de l'apprentissage allemand, du 26 avril 2006, régit cette profession réglementée et dresse la liste des différentes tâches de consultation et de soins aux patients, d'où ressort particulièrement l'importance des compétences sociales pour les personnes exerçant ce métier.

\section{Contextes d'analyse des compétences sociales : la recherche sur I'EFP et l'approche en psychologie}

Jusqu'au lendemain des débats des années 1980 et 1990, les compétences sociales étaient promues en tant qu'objectif formel de la formation initiale en milieu scolaire et en entreprise. La promotion des compétences sociales et interpersonnelles dans l'enseignement et la formation professionnelle initiale devait alors non pas se limiter à répondre aux seules exigences du monde du travail, mais bien permettre de faire émerger des citoyens adultes dotés d'un sens de la responsabilité individuelle. Le fondement normatif de cet objectif éducatif est inscrit dans la définition des compétences établie par le Conseil allemand de l'éducation en 1974, qui est aujourd'hui encore en vigueur pour l'EFP allemand.

À partir des années 1990, la recherche sur l'EFP s'est intéressée de manière plus exhaustive à l'importance des compétences sociales pour l'enseignement et la formation professionnels. Plusieurs aspects et dimensions de l'importance contextuelle de ces compétences ont été analysés dans un certain nombre d'études consacrées à des facteurs tels que les parcours professionnels et le développement personnel (Hoff, Lempert, Lappe, 1991, Grieger, 1994). D’autres travaux ont abordé des thématiques 
comme le jugement moral dans les métiers commerciaux notamment (Beck, Brütting, Lüdecke-Plümer, Minnameier, Schirmer, Schmidt, 1996 ; Beck \& al., 1998), les compétences de communication (van Buer, Matthäus, 1994, Wittmann, 2001) ou encore la capacité à travailler en équipe (Seyfried, 1995 ; Gomez, 2009).

Damm-Rüger \& Stiegler (1996) ont réalisé une analyse empirique des exigences en matière de compétences sociales dans des contextes et situations professionnels précis. Seyfried (1995), Euler \& Bauer-Klebl (2008), ainsi qu'Euler (2012) ont publié des travaux sur le concept de compétences sociales et les questions soulevées par leur promotion et leur évaluation. Ces auteur.es abordent le flou terminologique caractérisant la notion de compétences sociales, qui prend semble-t-il racine dans des points d'entrée théoriques et disciplinaires différents.

En psychologie par exemple, le concept de compétences sociales vise souvent la capacité d'une personne à s'adapter ou à adapter son comportement aux normes et aux règles sociales (Kanning, 2005). En revanche, les travaux sociologiques s'inscrivant dans le courant de la recherche-action considèrent que les compétences sociales sont davantage liées aux compétences d'interaction et de communication. Ils mettent l'accent sur le rôle de ces compétences pour coordonner l'interaction entre deux individus ou plus (Euler, Bauer-Klebl, 2008).

Si les définitions varient d'une discipline à l'autre, toutes s'accordent sur le fait que les compétences sociales sont déterminées par l'interaction entre plusieurs compétences et présentent, à ce titre, une architecture pluridimensionnelle (Schuler, Barthelme, 1999 ; Kanning, 2005 ; Euler, Bauer-Klebl, 2008). Quelles sont précisément ces compétences et quels termes employer pour les désigner et les distinguer les unes des autres? Cette question reste ouverte (Kanning, 2005).

L'absence de modèles et d'instruments adaptés pour mesurer et évaluer les compétences sociales a peu à peu suscité des critiques. Les méthodes en vigueur, telles que l'inventaire des compétences sociales de Kanning (2009,) se fondent essentiellement sur des conceptions génériques de ces compétences. Elles ne tiennent pas compte des exigences particulières des métiers en matière de compétences sociales et semblent peu ou pas du tout convenir à un usage en formation professionnelle ou aux fins du développement de compétences. Le manque de modèles propres à des métiers est donc un motif important qui explique qu'aujourd'hui encore, l'apprentissage et la formation ne sont que rarement adossés à des instruments de diagnostic systématiques et solides.

Le débat sur les compétences s'est renforcé avec le développement des cadres de certification en Europe, qui s'appuient sur une approche par les acquis d'apprentissage. Dans ce contexte, de nombreux travaux de recherche sur l'évaluation des acquis de l'apprentissage, y compris dans le champ de l'enseignement et de la formation professionnels (cf. Beck, Landenberger, Oser, 2016 ; Dietzen, Nickolaus, Rammstedt, Weiß, 2016), ont été menés. 
Ces travaux s'appuient sur la définition des compétences issue de la recherche empirique en éducation. Il s'agit d'une définition fonctionnaliste des compétences comme aptitudes à fournir une performance dans des types de situations et d'exigences données. Les compétences peuvent être décrites comme des savoirs, des savoir-faire, des stratégies, des habitudes ou des compétences-métier (Klieme, 2004 ; Hartig, 2008 ; Klieme, Hartig, 2007). Outre les particularités liées à son contexte, cette interprétation suppose des compétences pluridimensionnelles qui peuvent s'acquérir par l'apprentissage (Hartig, 2008).

À la différence de l'enseignement général, l'EFP prend pour point de référence le concept de compétence d'exercice professionnel (berufliche Handlungskompetenz) qui intègre les dimensions professionnelles, sociales et personnelles de la compétence, ainsi que les attitudes, les valeurs et les motivations (berufliche Handlungskompetenz). Ce concept est considéré comme un moyen au service du développement personnel des jeunes. Ceci impliquerait par conséquent que tout dispositif d'évaluation des compétences dans l'EFP se fonde sur ce concept, et que la compétence d'exercice professionnel soit envisagée de manière holistique. De telles exigences sont jugées irréalistes (Nickolaus, Seeber, 2013, p. 170).

C'est la raison pour laquelle le projet CoSMed, ${ }^{1}$ présenté ci-dessous, se propose d'emprunter une voie intermédiaire. Nous considérons que les compétences sociales sont des aptitudes pluridimensionnelles propres à un domaine ou à un métier qui s'acquièrent par l'apprentissage et sont modélisées et évaluées séparément des attitudes et des motivations. Nous aspirons à appréhender progressivement le concept de compétence d'exercice professionnel dans notre champ d'étude. En d'autres termes, nos travaux visent à ouvrir un accès empirique au champ des compétences sociales et à l'inscrire dans une conception large de la compétence d'exercice professionnel. Des relevés de données distincts permettront, en outre, d'identifier empiriquement des corrélations entre les compétences sociales et d'autres dimensions de la compétence professionnelle. Nous adoptons la définition de Kanning (2005, p. 155), qui considère comme socialement compétent "le comportement d'une personne dans une situation donnée, qui concourt à la réalisation de ses propres objectifs tout en étant socialement accepté».

Quel comportement répond à cette définition pour le métier d'assistant.e médical.e et quelles sont les compétences qui le facilitent ? Pour répondre à cette question, on

1. Le projet CoSMed - «Évaluer la compétence à partir de simulations et de tests adaptatifs en environnement médical ", a été financé par le ministère fédéral de l'Enseignement et de la recherche, entre novembre 2011 et mai 2015, dans le cadre du projet de recherche ASCOT : Évaluation de la compétence technologique dans l'enseignement et la formation professionnels. Tous les tests développés dans le cadre du projet ASCOT sont conçus comme des tests de performance utilisables également pour des évaluations à grande échelle. 
procède à une analyse du domaine ou des besoins. Elle révèle les exigences à satisfaire en matière de sociabilité et de communication, ainsi que les conditions générales dans lesquelles ces exigences sont satisfaites.

\section{Le modèle de compétences sociales dans le métier d'assistant·e médical·e}

La modélisation des compétences sociales et communicationnelles des assistant.es médicaux·ales, au sein du projet CoSMed, se fonde sur une analyse détaillée des besoins, comprenant une étude de documents (tels que les règlements d'apprentissage, les programmes, les épreuves d'examen), une analyse par le biais de discussions collectives avec des praticien-nes spécialistes et treize entretiens semi-directifs auprès d'apprenti·ess, de médecins et d'assistant.es médicaux·ales ayant l'expérience du métier. L'évaluation de 1800 offres d'emploi, publiées par l'Agence fédérale pour l'emploi, a complété cette analyse.

L'analyse des besoins révèle qu'une multitude d'indicateurs caractérise le quotidien de travail des assistant.es médicaux.ales. Leurs partenaires d'interaction sont, d'une part, les patient.es et les membres de leurs familles, d'autre part, les collègues de l'équipe du cabinet avec différents niveaux hiérarchiques (médecins, assistant·es médicaux·ales et aprenti.e.s). Elles/Ils interagissent également, bien que plus rarement, avec des partenaires issus d'autres cabinets, de laboratoires et d'entreprises commerciales. L'assistant.e médical.e joue souvent un rôle d'intermédiaire entre la.le médecin et ses patient.es. C'est le premier interlocuteur au téléphone et à l'accueil. Outre les tâches d'organisation, elle/il a pour mission de déceler les inquiétudes et d'identifier l'état de santé des patient.es afin d'évaluer le degré d'urgence du cas. Un niveau de stress élevé et des risques de conflit sont inhérents à ces fonctions, notamment lorsque les patients doivent attendre ou sont particulièrement tendus (craintes pour leur santé, peurs, douleur, contrainte de temps). Les missions de l'assistant.e médical.e comportent également des fonctions médicales, comme la réalisation d'examens préliminaires (y compris les prises de sang ou les électrocardiogrammes). Lorsqu'elles/ils réalisent ce type d'actes, il arrive qu'elles/ils aient besoin d'expliquer les étapes de la procédure de manière claire ou de rassurer les patient.es.

Cependant, des situations très exigeantes sur le plan social et de la communication peuvent également survenir entre membres de l'équipe du cabinet médical. Par exemple, lorsqu'il s'agit de convenir des domaines de responsabilité des un.es et des autres, de coordonner les périodes de congés ou des rapports avec les supérieur-es hiérarchiques. Les conditions de travail, comme la charge de travail, les contraintes de 
temps, la transparence et l'équité de la répartition des fonctions, ainsi que l'ambiance générale de travail peuvent atténuer ou renforcer ces exigences.

En complément de ces observations empiriques des tâches des assistant.es médical.es et des exigences qui pèsent sur elles/eux, un travail théorique permet d'identifier les dimensions des compétences sociales nécessaires pour répondre aux exigences repérées et de les transposer en un modèle illustré par le graphique $\mathbf{1}$.

\section{Graphique 1. Modèle de compétences sociales pour les assistant·es médicaux·ales du projet CoSMed}

- avec les patients et leur proches avec les membres de l'équipe

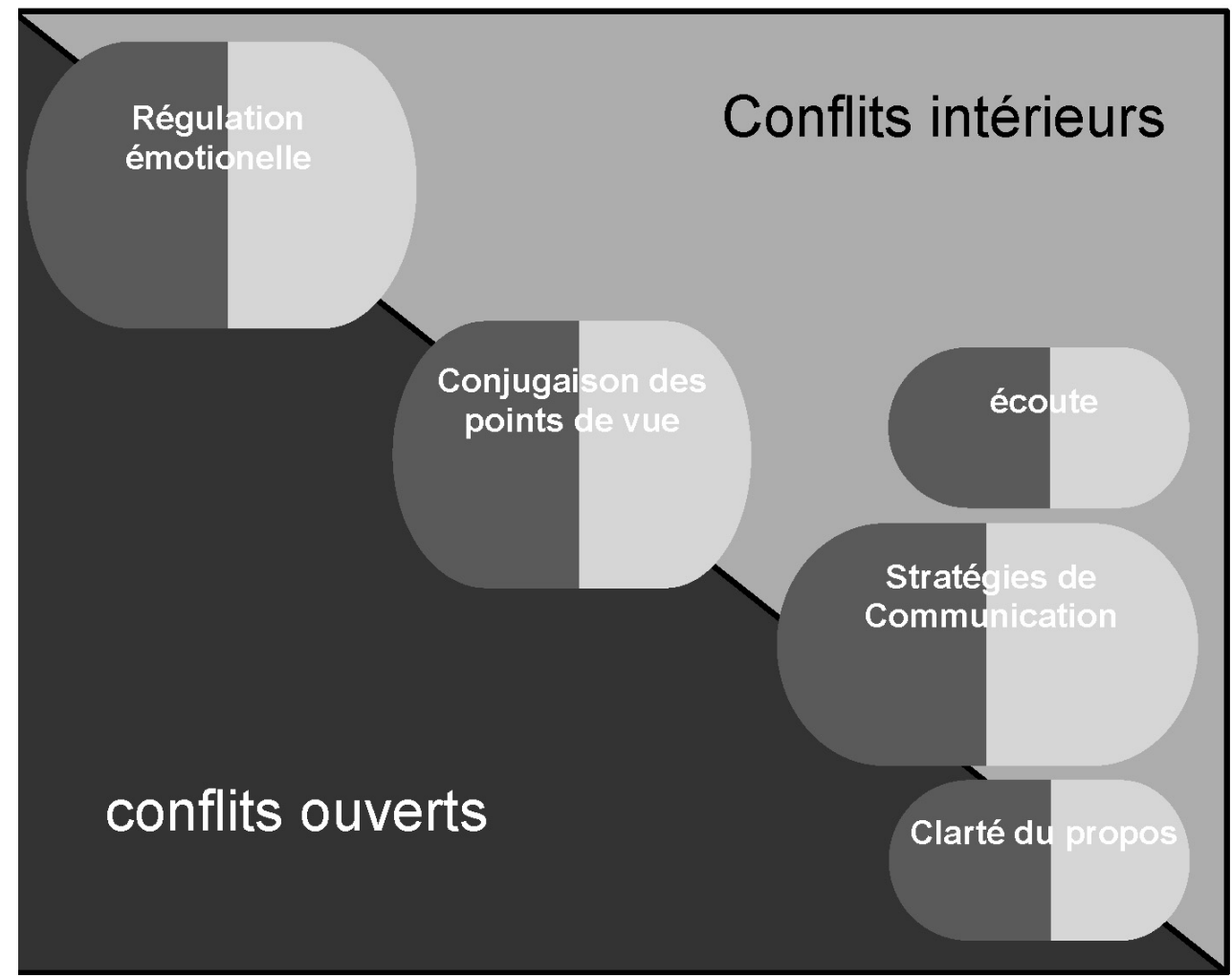

Source : Auteures. 
La première compétence sociale essentielle au métier d'assistant·e médical·e est la "régulation émotionnelle ». Elle suppose d'exercer un contrôle intérieur sur ses émotions, telles que la peur, la honte, le dégoût ou même une gaieté déplacée, et d'être capable d'avoir des réactions adaptées vis-à-vis de l'extérieur. Un·e assistant·e médical·e compétent.e ne laisse pas libre cours à ses émotions, pas plus qu'elle ne peut en faire abstraction ou les annihiler.

Concernant l'opérationnalisation de cette compétence, nous nous sommes intéressées à plusieurs stratégies de régulation émotionnelle (par exemple, Gross, 2009), dont la stratégie de "mise en acte de décharge" (exprimer extérieurement ses émotions sans filtre), "réprimer l'expression de l'émotion" (élimination superficielle de l'émotion) et "modification de la situation" (les émotions sont profondément modifiées après une réévaluation cognitive de la situation déclencheuse). Des stratégies visant à produire une expression du ressenti adaptée à la situation sont également employées et nous prenons en compte leurs conséquences respectives : "jeu en profondeur " (deep acting) et "jeu superficiel» (surface acting), Hochschild, 1983 ; Giardini, Frese, 2006). Le " jeu en profondeur " opère une réelle transformation des émotions afin de réduire la discordance perçue entre les émotions d'une personne et les exigences de son environnement. Le « jeu superficiel » altère uniquement l'expression externe de l'émotion. Cela signifie que l'émotion initiale perdure, ainsi que la discordance. Il est prouvé que des discordances fréquentes entre la perception intérieure et l'expression extérieure peuvent accroître le risque de problèmes de santé (Zapf, 2002). Cependant, puisque le "jeu en profondeur " est plus difficile à mettre en œuvre, il a moins de chances d'être appliqué, notamment dans des situations où le niveau de stress ressenti est fort.

Pour opérationnaliser la "régulation émotionnelle " au sein du projet CoSMed, nous avons choisi des stratégies applicables, réalistes et souhaitables pour la profession d'assistant.e médical.e. Ces stratégies suivent un continuum allant de "sur-adapté " à « impulsif ", en passant par "régulation équilibrée ". L'emploi de stratégies visant à opérer un changement réel du ressenti intérieur, et par conséquent à réduire les discordances sans être ni trop impulsif, ni suradapté, est qualifié de comportement particulièrement compétent. En revanche, réprimer purement ses émotions ou les exprimer sans filtre est répertorié comme mauvais pour la santé sur le long terme et n’aboutit pas à la résolution de conflit. Le fait d'utiliser ces stratégies est donc jugé comme un comportement moins compétent.

La deuxième compétence sociale essentielle d'un·e assistant.e médical.e est celle consistant à " conjuguer les points de vue ". Pour être socialement compétent, un.e assistant.e médical.e doit être à même de comprendre les points de vue, les émotions et les besoins de ses interlocuteurs, même dans des situations difficiles, tout en gardant parfaitement à l'esprit les besoins du cabinet. Ce faisant, il/elle doit articuler les divers points de vue de la manière la plus constructive possible. Interviennent ici les apports théoriques suivants : le modèle de conjugaison des points de vue sociaux, éla- 
boré par Selman (2003) et l'adaptation allemande de sa démarche par Mischo (2004). Selman (2003) formule l'hypothèse d'un modèle de développement sociocognitif en cinq stades, où chaque passage à un stade supérieur est marqué par la structuration, la distinction et la prise en compte d'un plus grand nombre de points de vue ${ }^{2}$.

Les "stratégies de communication " compétentes constituent la troisième compétence sociale essentielle du modèle. Un.e assistant.e médical.e compétent.e oriente la communication vers une solution satisfaisante pour les deux parties, même dans des situations où la conversation est difficile, et sait donner à ses interlocuteurs-trices le sentiment d'être compris et pris au sérieux. Le cadre d'évaluation choisi pour ce projet est dérivé des résultats des relevés de données initiaux et s'appuie sur plusieurs théories (dont celles proposées par Schulz von Thun, 2004 et Rosenberg, 2006). L'évaluation met en avant le contenu et les dimensions méta-communicationnelles. On y demande, notamment, s'il existe des explications objectives ou des types de réaction à la critique. Le but est aussi de prendre en compte le ton de voix employé.

Le modèle des compétences sociales des assistant.es médicaux.ales comprend deux autres dimensions : la "clarté du propos" et "l'écoute "3. Le volet "clarté du propos" couvre les éléments de théorie de la communication, tels que la simplicité, la brièveté et la concision du message (Langer, Schulz von Thun, Tausch, 1999, p. 15 sq.). Le volet "écoute " aborde la réception et le décodage du message reçu, par exemple l'inquiétude exprimée par un.e patient.e ou un·e proche.

Les compétences sont placées sur un fond gris clair et un fond gris foncé dans le graphique 1. Ceci reflète la distinction entre les situations de conflit intérieur et de conflit ouvert (Glasl, 2002). Les conflits ouverts sont des situations de différend réel avec une autre personne, par exemple une plainte d'un.e patient.e. Les conflits intérieurs sont les situations où l'assistant.e médical·e est en conflit " avec elle/lui-même ", par exemple lorsqu'elle/il est contraint.e de jongler entre plusieurs tâches urgentes.

Chaque compétence est elle-même divisée en une zone noire, qui représente l'interaction avec les patients et leurs proches, et une zone blanche, qui illustre l'interaction

2. Le modèle de Selman repose sur les idées de Piaget sur le développement cognitif (cf. Piaget 1973, 2003). Au premier stade du modèle de Selman, l'individu perçoit les interactions sociales à travers un prisme égocentré et se concentre sur ses intérêts personnels. À mesure qu'il passe aux stades suivants, il devient capable de percevoir différents points de vue en même temps et de les comprendre plus en profondeur. L'individu apprend à voir le monde en adoptant le point de vue d'autres personnes présentes et à inclure des intérêts différents au tableau global. Le dernier stade du modèle est caractérisé par une vision d'ensemble ancrée dans un système de valeurs et de normes morales qui peuvent transcender les intérêts personnels des personnes présentes.

3. La compétence de clarté du propos désigne la capacité du locuteur à s'adapter au langage et aux connaissances du destinataire afin qu'il puisse comprendre l'argument exposé. La compétence d'écoute comprend des techniques permettant à la personne de mieux comprendre le contenu qu'elle écoute, par exemple en reformulant, en résumant ou en posant des questions. 
avec les collègues de l'équipe. Dans ce dernier cas, les compétences peuvent se manifester différemment selon l'intensité, la durée et le degré de formalisme des relations.

Le modèle de compétence a été évalué au moyen d'un questionnaire soumis à 27 spécialistes praticien-nes, les critères étant la référence à la réalité, la pertinence et l'exhaustivité. Toutes les dimensions de compétence ont été évaluées sur une échelle de un à quatre (quatre $=$ totalement d'accord). Si les réponses collectées se situaient toutes entre trois et quatre sur l'échelle, nous avons néanmoins pu réunir de nombreuses propositions d'amélioration. En outre, les situations de test ont été évaluées par les praticien-nes spécialistes et les aprenti.e.s grâce à un questionnaire en ligne portant sur l'authenticité, la pertinence et le caractère compréhensible des situations de test. La validation du contenu par des représentant.es de la pratique est l'un des principaux critères de conception des évaluations en situation. La phase suivante du travail de recherche a consisté à utiliser le modèle de recherche pour développer un instrument de test des compétences sociales des assistant·es médicaux·ales.

\section{Validation du modèle par un test vidéo de jugement en situation}

Comme nous l'avons observé ci-dessus, les recherches menées jusqu'à présent en matière de compétences sociales démontrent que les compétences sociales sont fortement tributaires de leur contexte, de sorte qu'il est quasiment impossible de trouver une définition générale qui nous dispenserait d'évaluations non spécifiques des compétences. Réduire le contexte à un nombre raisonnable de facteurs d'influence, par exemple ceux qui découlent d'un rôle professionnel bien défini, ouvre la voie à l'analyse quantitative. Notre test visait donc une évaluation contextuelle des trois compétences sociales essentielles des assistant.es médicaux·ales, soit la "régulation émotionnelle", "la conjugaison des points de vue " ${ }^{4}$ et les "stratégies de communication ". Les instruments du test n'incluent pas la clarté du propos ni l'écoute, car ces aspects oraux se prêtent peu au format du test écrit.

Pour répondre à l'exigence contextuelle, le test a été conçu sous forme de simulation de jugement en situation (Lievens, Chan, 2010 ; Kanning, Schuler, 2014). Les tests de jugement en situation (TJS) (Situational Judgment Test (SJT) sont une méthode, redécouverte ces dernières années, permettant de mesurer les compétences sociales et émotionnelles. Les auteures l'ont jugée tout à fait adaptée à la modélisation des compétences sociales-métier. Le principe du TJS consiste à demander à des personnes d'évaluer des scénarii comportementaux fictifs dans différents types de situations pro-

4. NdT : soit la capacité à adopter différents points de vue. 
fessionnelles ou à leur demander de classer les différents comportements possibles du plus efficace au moins efficace.

Le TJS, développé par le projet CoSMed pour mesurer les compétences sociales, comprend une présentation vidéo d'un cabinet médical fictif et douze scènes vidéo de situations critiques. Chacune des douze séquences débute par un bref texte introductif. La séquence 1 s'ouvre par exemple sur le texte suivant : "Un patient en urgence vient d'arriver au cabinet et passe en premier avant les autres patients. "

Les participant.es visionnent ensuite une vidéo dans laquelle des différents interlocuteurs (patients, proches, médecins ou collègues) parlent directement à la caméra. Les prises de parole directes à la première personne accentuent le réalisme de la situation. Ce procédé atténue l'identification aux locuteurs et renforce le degré d'implication émotionnelle.

Les douze scénarii ont été choisis afin de présenter les besoins ou exigences des patient.es en situation à la réception et dans la salle de consultation. Les situations impliquant l'équipe médicale se déroulent dans des espaces tels que la salle d'équipe ou le couloir. Six scénarii abordent des conflits ouverts et six autres des conflits intérieurs. Après chaque vidéo, les participants doivent répondre à des questions mesurant les trois compétences sociales essentielles. Différents formats de réponse ont été testés lors de la phase pilote, afin de saisir au plus près les exigences pour chaque compétence. Dans la version finale du test, nous avons opté pour des questions sous forme de texte avec réponses ouvertes et réponses à choix multiples, ainsi que des réponses graphiques.

\subsection{Collecte de données et échantillonnage}

Le module de test a été utilisé lors de trois phases de collecte de données, auxquelles ont participé au total $\mathrm{N}=901$ aprenti·e.s assistant·es médicaux·ales en troisième année d'apprentissage. Tous les relevés de données ont été effectués en salle de classe dans des centres de formation pour apprentis de Rhénanie du Nord-Westphalie, de RhénaniePalatinat et de Berlin, sur les ordinateurs des écoles et en présence de membres du $\mathrm{BBIB}$ travaillant sur le projet CoSMed et qui donnaient les instructions. Au préalable, les aprenti.e.s ont reçu une lettre d'information leur expliquant la nature et les objectifs de ce relevé de données, la participation au test était anonyme et volontaire.

Les deux premiers relevés de données ont été utilisés durant la phase pilote de l'instrument de test. Ils visaient essentiellement à vérifier non pas la performance des participant.es, mais bien la qualité de l'instrument, notamment son contenu et son respect des critères théoriques. La première phase pilote s'est achevée au début de l'année 2013, avec $\mathrm{N}=236$ aprenti.e.s. La deuxième phase pilote a démarré durant l'hiver 2013/2014 et N = 260 aprenti.e.s y ont participé. Les descriptions détaillées de 
ces deux échantillons ne sont pas présentées ici, mais elles sont comparables à l'échantillon collecté pour l'enquête principale décrite ci-dessous.

L'enquête principale s'est déroulée en 2014, avec la participation de $\mathrm{N}=405$ aprenti.e.s en troisième année d'apprentissage. L'âge moyen de la cohorte était de 21,3 ans, soit légèrement plus jeune que la moyenne de la cohorte 2013 en Allemagne, qui avoisine les 22,7 ans pour ce métier, comme le précisent les statistiques de l'EFP (BIBB, 2014). $98,7 \%$ des aprenti.e.s répondant au test étaient des femmes ; ce chiffre est rigoureusement identique à celui de la représentation féminine de la cohorte entière de 2013 (BIBB, 2013). La répartition du niveau de qualification de l'échantillon, à la sortie du système scolaire, était similaire à celle de la cohorte entière de 2013, avec toutefois une plus forte représentation des niveaux de qualification supérieurs en fin de scolarité (voir tableau 1). Ceci tient probablement au fait que les établissements ont tendance à présenter leurs classes les plus performantes pour participer à ce type d'enquête. Pour des raisons de coût et d'efficacité, le relevé de données a été effectué à partir d'un échantillon de convenance et mené dans trois Länder (États fédérés) allemands seulement. En dépit de ces restrictions, la transférabilité des résultats à d'autres aprenti-e.s assistant·es médicaux-ales en troisième année de formation est jugée bonne du fait de la composition démographique.

Tableau 1. Détails de l'échantillon et de la cohorte

\begin{tabular}{|l|c|c|}
\cline { 2 - 3 } \multicolumn{1}{c|}{} & Échantillon & Cohorte $\mathbf{2 0 1 3}$ \\
\hline A quitté le système scolaire sans qualification & $1.6 \%$ & $1,2 \%$ \\
\hline Enseignement secondaire court & $14.4 \%$ & $19.0 \%$ \\
\hline Premier cycle d'enseignement secondaire & $53.5 \%$ & $65.5 \%$ \\
\hline $\begin{array}{l}\text { Diplôme de fin d'enseignement secondaire donnant accès à } \\
\text { l'enseignement supérieur }\end{array}$ & $30.6 \%$ & $14.2 \%$ \\
\hline Pays d'origine: Allemagne & $87.8 \%$ & 90.7 \\
\hline
\end{tabular}

Source : Auteures.

\subsection{Résultats empiriques : bilan des compétences sociales des assistante.s médicaux·ales}

Avant de présenter les niveaux de compétence des assistant·es médicaux·ales, nous devions vérifier que la procédure d'évaluation utilisée fonctionnait correctement et permettait bien de décrire les dimensions de compétence visées. Les procédures de vérification sont détaillées dans l'encadré 2 . Le modèle de compétence ayant enregistré le meilleur résultat au test empirique est illustré par le graphique 2. Dans ce modèle, les dimensions de la compétence que sont la "régulation émotionnelle » et les "stratégies de communication" présentent respectivement des sous-ensembles de dimensions, ce 
qui indique que les exigences varient probablement en fonction des types de situations : conflits intérieurs (par exemple une situation gênante) ou extérieurs (conflit de répartition), conflits avec les patients (par exemple un mauvais diagnostic) ou au sein de l'équipe (par exemple du harcèlement) ; on observe par ailleurs un autre paramètre de variation, lié à l'émotion perçue (par exemple de la colère).

Pour la dimension " conjuguer les points de vue " en revanche, les caractéristiques de la situation ne semblent pas générer de différence ; c'est le domaine auquel le modèle unidimensionnel se prête le mieux. Tirons les conséquences de ce résultat : pour cartographier le quotidien de travail des assistant.es médicaux.ales, il convient de prendre en compte les émotions suscitées comme si elles constituaient une caractéristique supplémentaire venant s'ajouter aux deux caractéristiques contingentes (de la situation).

Les coefficients d'analyse mettent en évidence une forte corrélation entre les compétences dans deux cas. La compétence "stratégies de communication " est en interrelation avec les deux autres compétences. Une corrélation forte de coefficient 0,67 existe entre les compétences "conjuguer les points de vue " et "stratégies de communication". Les personnes qui parviennent à avoir une représentation plus complexe de la situation sociale, tout en adoptant un grand nombre de points de vue différenciés, sont donc les plus compétentes pour appliquer les «stratégies de communication ». Une corrélation de coefficient 0,39 est établie entre la compétence "régulation émotionnelle " et " conjuguer les points de vue».

En revanche, le modèle ne permet pas d'établir de corrélation entre les compétences "régulation émotionnelle " et " conjuguer les points de vue ". Cela signifie que les personnes les plus compétentes pour réguler leurs émotions n’étaient pas nécessairement les plus à même d'articuler différents points de vue. 


\section{Graphique 2. Analyse factorielle confirmatoire (CFA) du modèle de compétences sociales et communicationnelles des assistant·es médicaux·ales}

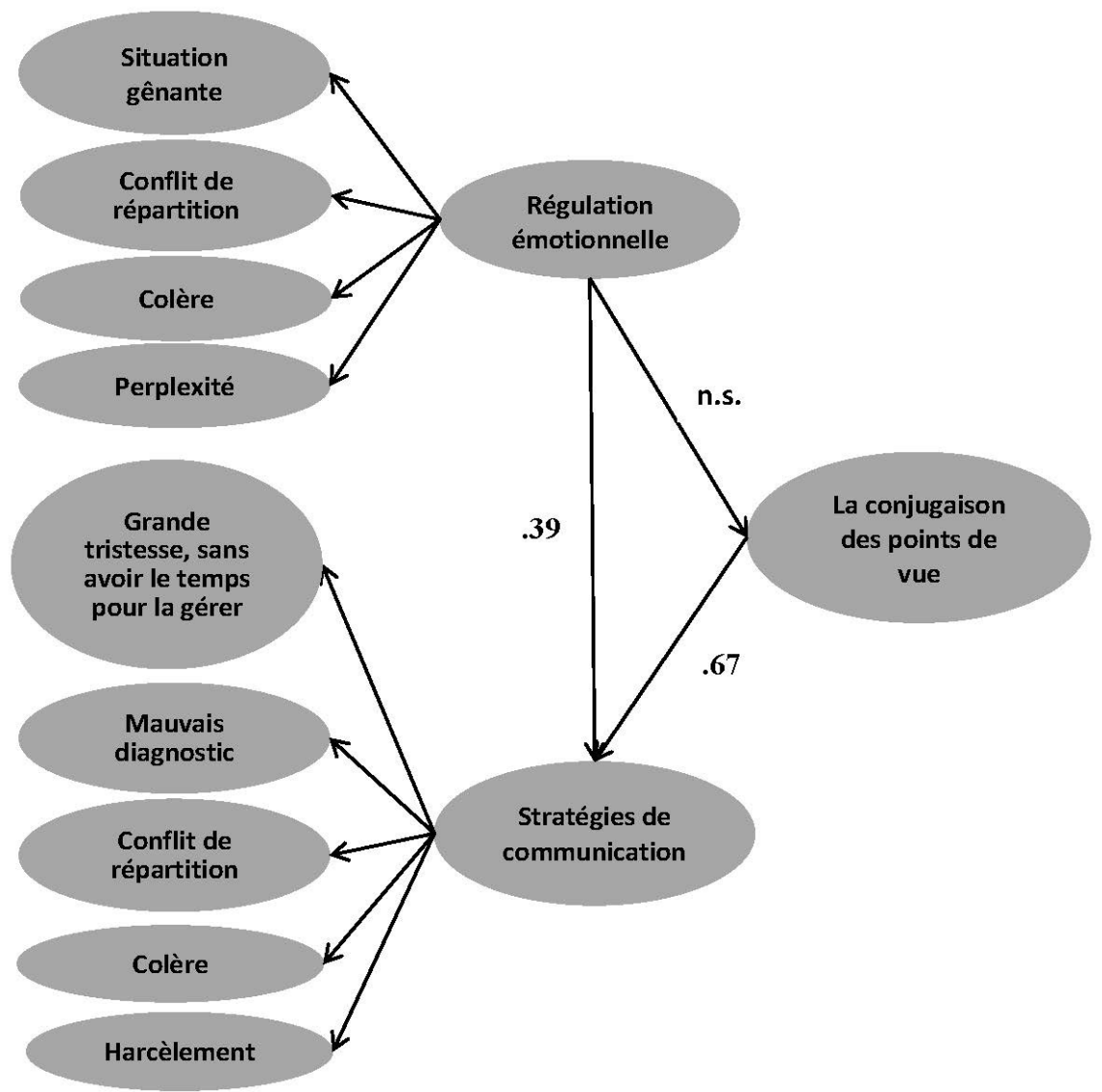

Ce modèle montre les trois principales dimensions des compétences, leurs interrelations et les sous-ensembles qui les structurent. Les rapports entre " stratégies de communication » et « conjuguer les points de vue » $(r=0,67)$, d'une part, et entre « stratégies de communication » et « régulation émotionnelle » $(r=0.39)$, d'autre part, sont tous deux très significatifs $(p<0,001)$.

Mesures de l'ajustement du modèle : $\mathrm{X} 2=636,658, \mathrm{df}=554$; indice d'adaptation relative (Comparative Fit Index) $(\mathrm{CFI})=0,96$; moyenne quadratique de l'erreur d'approximation (Root Mean Square Error of Approximation) (RMSEA) =0,02 et résiduel standardisé de moyenne quadratique (Standardized Root Mean Square Re sidual $($ SRMR $)=0,07$.

Source : Auteures. 


\section{Encadré 2. Qualité du modèle}

Pour vérifier la qualité de nos instruments, dans un premier temps, nous avons analysé la cohérence interne (ou fiabilité des échelles) avec un résultat satisfaisant à bon (coefficient de Cronbach $\alpha=0,87$ pour la « régulation émotionnelle »; $\alpha=0,75$ pour « conjuguer les points de vue » et $\alpha=0,68$ pour les «stratégies de communication »).

Dans un deuxième temps, nous avons examiné les données en nous demandant si elles confirment le postulat d'une structure tridimensionnelle des compétences sociales des assistant.es médicaux·ales. La méthode choisie pour ce faire a été l'analyse de facteurs de confirmation (CFA), car les indices d'adaptation de la CFA révèlent les similitudes entre la structure des données et les modèles postulés. Nous avons comparé un modèle unidimensionnel et plusieurs modèles pluridimensionnels présentant tous la structure tridimensionnelle postulée. Nous avons utilisé différents modèles de mesure pour chacune des trois dimensions de compétence. Le modèle offrant la description la plus fidèle de la structure des données est illustré par le graphique $\mathbf{2}$.

Les résultats du test révèlent des écarts de performance considérables entre les participant.es, ainsi que d'importantes lacunes dans les trois domaines de compétence. Ainsi, pour la compétence "régulation émotionnelle ", on observe, dans les douze situations testées (tests $\mathrm{t}$, valeur $\mathrm{p}<0,001$ ), de très grands écarts entre l'intensité de l'émotion ressentie et celle exprimée, appelés discordance émotionnelle. Pour passer outre cette discordance, la majorité des aprenti.e.s se contentent de dissimuler leur véritable ressenti et d'adopter ce que l'on appelle un "jeu superficiel» (Hochschild, 1983). Les personnes testées recourent davantage au jeu superficiel en situation avec des patient.es qu'avec des collègues. Ceci indique que dans leur quotidien de travail, les assistant·es médicaux·ales reprennent à leur compte la formule " restez aimable et souriez », un adage souvent brandi en étendard dans les métiers du secteur des services, et qu'elles/ils sont plus enclin·es à l'appliquer visà-vis des patient.es que face à leurs collègues. Toutefois, une telle démarche présente un risque élevé, car les interactions peuvent être vécues à court terme comme moins authentiques. Le risque qui pèse à long terme sur les assistant.es médical-es/aux est qu’elles/ils deviennent blasé.es ou rencontrent des problèmes de santé. Sur l'échantillon testé, le jeu superficiel avait cinq fois plus de chances d'être utilisé qu'une stratégie de réduction de la discordance émotionnelle par réévaluation consciente et réinterprétation de la situation. Cependant, la réévaluation/réinterprétation est classée comme une stratégie plus compétente dans le modèle, car elle entraîne des effets positifs à la fois sur la personne qui régule ses émotions et sur la/le partenaire d'interaction. Pour les douze scènes testées, la réévaluation positive figurait en dernier ou avant-dernier choix de stratégie.

La dimension " conjuguer des points de vue " ouvre elle aussi des perspectives de développement aux aprenti.e.s. Environ un tiers des aprenti.e.s sont capables de prendre en compte les points de vue de toutes les parties prenantes et d'analyser en détail au moins un des points de vue, plutôt que de se limiter au point de vue d'une seule partie (par exemple soi-même). Il n'en reste pas moins, et c'est le revers de la médaille, que deux tiers 
des participant.es n'en sont pas capables. Ross (1977) a qualifié " d'erreur fondamentale d'attribution" la tendance qu'ont les personnes à chercher des causes extérieures à leur propre comportement et à attribuer le comportement d'autrui à des facteurs internes. Cette tendance s'est également manifestée sur notre échantillon, notamment dans le cas des conflits ouverts. En moyenne, les participant.es sont mieux parvenu.es à approfondir la représentation de leur propre point de vue que celui d'autres participant.es. Un seul sous-groupe a régulièrement mis de côté son point de vue pour s'intéresser à celui de ses interlocuteurs. Un tiers des participant-es ne sont pas parvenu-es à progresser au-delà du niveau de compétence le plus faible, caractérisé par le fait d'incriminer systématiquement la partie opposée (par exemple : "Ce patient n'est qu'un impatientlest complètement impossible à raisonner/n'est qu'un idiot»). En revanche, le tableau est bien différent pour les situations de conflits intérieurs où la part des aprenti-e.s qui négligent leur propre point de vue est bien plus élevée.

Seulement $20 \%$ des aprenti.e.s atteignent le meilleur niveau de compétence, celui où l'on aborde les situations avec une vue d'ensemble, et cela concerne uniquement des situations individuelles. Il ressort des résultats de l'enquête que l'intensité du ressenti d'une personne peut légèrement diminuer ses performances dans le domaine « conjuguer les points de vue ". Ceci souligne une fois de plus à quel point il est crucial de savoir réguler efficacement ses émotions personnelles.

Pour la compétence "stratégies de communication", les participant-es donnent quasiment tous.tes les mêmes réponses sur le sujet " conserver un ton aimable en toutes circonstances ». Dans quelques cas exceptionnels, des participant.es ont validé des réponses dont l'intonation seule était impolie. En revanche, les différences apparaissent dès lors qu'il s'agit de trouver sa propre formulation pour donner une impression authentique d'assurance et de réconfort, sans employer les habituelles phrases toutes faites. Les aprenti.e.s affichent des taux de réussite variables pour les tâches impliquant d'éclaircir et d'expliquer des situations et de transmettre des informations pertinentes. Certain.es s'efforcent de proposer une solution à leur interlocuteur, tandis que d'autres n'y parviennent pas. Dans certains cas, les participant.es en situation de conflit intérieur sortent de leur rôle professionnel, soit en prenant pour elles/eux les agressions verbales des patient.es, soit en tentant de se justifier.

Dans l'ensemble, les résultats du test montrent que les aprenti.e.s ont besoin d'approfondir et de développer les trois dimensions de compétence. Pourtant, à ce jour, l'encouragement systématique de ces compétences est loin de constituer un objet de formation systématique. Le modèle de compétences sociales pour les assistant·es médicaux·ales constitue un point de départ à cet effort de formation, car tous les éléments du modèle s'appuient sur un arsenal théorique déjà mobilisé dans d'autres contextes pour développer des formations (pour une vue d'ensemble, voir Tschöpe, Monnier, 2016). 


\section{Conclusion}

La démarche de modélisation et d'évaluation des compétences sociales dans l'enseignement et la formation professionnels (EFP), présentée dans cet article, s'inscrit dans la logique de compétences devant être acquises, pluridimensionnelles et propres à un contexte. Conformément à la définition de Kanning (2003), les compétences sociales permettent à une personne d'adapter son comportement à des situations particulières pour concourir à la réalisation de ses propres objectifs, tout en adoptant un comportement socialement acceptable.

Le contexte est bel et bien précisé dans notre étude, puisqu'elle se centre sur le métier d'assistant.e médical.e en apprentissage, une profession très prisée par les jeunes femmes en Allemagne, qui présente de réels défis en matière d'interaction sociale. Partant des exigences propres à ce métier et de situations sociales types, nous avons développé un modèle de compétence composé des trois grandes compétences que sont la "régulation des émotions ", la capacité à " conjuguer les points de vue " et les "stratégies de communication ". Les résultats de notre étude révèlent que seul un petit nombre d'aprenti-e.s obtient le niveau de compétence requis. Ceci a pour corollaire un risque important de malentendus et d'agacements lors des interactions, ainsi que de perturbations de l'état de santé des aprenti.e.s.

Les résultats de la modélisation et l'évaluation des compétences sociales sont tout aussi pertinents pour la recherche que pour la pratique de l'EFP. Du point de vue de la recherche, le travail effectué permet de clarifier l'architecture des compétences sociales, ainsi que leurs caractéristiques contextuelles ou circonstancielles. Il permet également de favoriser le développement de procédures d'évaluation des compétences sociales conçues sur mesure pour des métiers précis.

Concernant la pratique, ces conclusions pourraient, par exemple, être utilisées pour élaborer les règlements d'apprentissage, les programmes, et pour agencer la structure des examens. La promotion ciblée de compétences sociales pour des métiers précis représente également un champ de transfert important de ces résultats.

Pour conclure, il existe un besoin urgent et manifeste d'actions ciblées de promotion et d'encouragement des compétences sociales durant la formation en apprentissage des assistant.es médicaux.ales. Lobjectif est double : faciliter les comportements professionnels face aux patients, d'une part, et permettre l'adoption de démarches pérennes et satisfaisantes sur le plan personnel, d'autre part. Il s'agit en effet de faire face à des situations critiques dans un métier socialement très exigeant. Le déficit de recherche en la matière a été souligné de longue date. Les modèles de compétences sociales propres à un métier constituent un point de départ qui révèle la nature des compétences à intégrer dans la formation et les modalités précises de formation afin de préparer les apprentis aux difficultés qu'ils rencontreront dans leurs interactions. 


\section{Bibliographie}

Baethge M., Achtenhagen F., Arends L., Babic E., Baethge-Kinsky V., Weber S. (2006), Berufsbildungs-PISA - Machbarkeitsstudie, Stuttgart, Franz Steiner.

Baethge M., Seeber S. (2016), „Die gemeinsame theoretische und methodische Basis der ASCOT-Projekt", in Beck K., Landenberger M., Oser F. (eds.) (2016), Technologiebasierte Kompetenzmessung in der beruflichen Bildung: Ergebnisse aus der BMBF-Förderinitiative ASCOT, Wirtschaft - Beruf - Ethik n 32, Bielefeld, W. Bertelsmann, pp. 15-33.

Beck K., Brütting B., Lüdecke-Plümer S., Minnameier G., Schirmer U., Schmid N.S. (1996), „Zur Entwicklung moralischer Urteilskompetenz in der kaufmännischen Erstausbildung - Empirische Befunde und praktische Probleme“, Zeitschrift für Berufsund Wirtschaftspädagogik, Beiheft $\mathrm{n}^{\circ} 13$, pp. 187-206.

Beck K., Bienengräber T., Heinrichs K., Lang B., Lüdecke-Plümer S., Minnameier G., Parche-Kawik K., Zirkel A. (1998), „Die moralische Urteils- und Handlungskompetenz von kaufmännischen Lehrlingen - Entwicklungsbedingungen und ihre pädagogische Gestaltung“, in Beck K., Dubs R. (eds.), Kompetenzentwicklung in der Berufserziehung, kognitive, motivationale und moralische Dimensionen kaufmännischer Qualifizierungsprozesse, Stuttgart, Franz Steiner.

Beck K., Landenberger M., Oser F. (eds.) (2016), Technologiebasierte Kompetenzmessung in der beruflichen Bildung: Ergebnisse aus der BMBF-Förderinitiative ASCOT, Wirtschaft - Beruf - Ethik, n 32, Bielefeld W. Bertelsmann.

Bühner M. (2011), Einführung in die Test- und Fragebogenkonstruktion, München, Pearson Studium.

Damm-Rüger S. (1996), Soziale Qualifikation im Beruf: Eine Studie zu typischen Anforderungen in unterschiedlichen Tätigkeitsfeldern, Bielefeld, W. Bertelsmann.

Deutscher Bildungsrat (1974), Empfehlungen der Bildungskommission zur Neuordnung der Sekundarstufe II. Konzept für eine Verbindung von allgemeinem und beruflichem Lernen, Bonn, Deutscher Bildungsrat.

Dietzen A., Nickolaus R., Rammstedt B., Weiß R. (eds.) (2016), Kompetenzorientierung: Berufliche Kompetenzen entwickeln, messen und anerkennen, Bielefeld, W. Bertelsmann.

Dietzen A., Monnier M., Tschöpe T. (2012), Soziale Kompetenzen von medizinischen Fachangestellten messen - Entwicklung eines Verfahrens im Projekt CoSMed, BWP 6/2012, pp. 24-28.

Euler D., Bauer-Klebl A. (2008), „Präzisierungen: Bestimmung von Sozialkompetenzen als didaktisches Konstrukt", in Euler D. (ed.), Sozialkompetenzen in der beruflichen Bildung. Didaktische Förderung und Prüfung, Bern, Haupt. 
Euler D. (2012), „Von der programmatischen Formel zum didaktischen Konzept: Sozialkompetenzen präzisieren, fördern und beurteilen“, in Niedermair G. (ed.), Kompetenzen, entwickeln, messen und bewerten, Linz, Trauner, pp. 183-199.

Giardini A., Frese M. (2006), "Reducing the negative effects of emotion work in service occupations: Emotional competence as a psychological resource", Journal of Occupational Health Psychology, $\mathrm{n}^{\circ}$ 11(1), pp. 63-75.

Glasl F. (2002), Konfliktmanagement: Ein Handbuch für Führungskräfte, Beraterinnen und Berater, Bern, Freies Geistesleben.

GomézJ.M. (2009), „Problem-und aufgabenorientierteFörderungvonTeamkompetenzen - Eine empirische Studie“, Zeitschrift für Berufs- und Wirtschaftspädagogik, n ${ }^{\circ} 105$, pp. 378-405.

Grieger D. (1994), „Die Entwicklung von fachübergreifenden Qualifikationen während der Ausbildung", in Dybowkski G. (ed), Lernen heute - Fragen für morgen. Zur Lernforschung in der Berufsbildung. Berichte zur beruflichen Bildung, Heft 168. Bielefeld, W. Bertelsmann, pp. 137-150.

Gross J.J. (2009), Handbook of emotion regulation, New York, Guilford Press.

Hartig J. (2008), „Kompetenzen als Ergebnisse von Bildungsprozessen“, in Jude N., Hartig J., Klieme E. (eds.), Kompetenzerfassung in pädagogischen Handlungsfeldern. Theorien, Konzepte und Methoden, Bonn, BMBF, pp. 15-26.

Hochschild A.R. (1983), The managed heart, Los Angeles, University of California Press.

Hoff F.H., Lempert W., Lappe L. (1991), Persönlichkeitsentwicklung in Facharbeiterbiographien: Eine Längsschnittstudie, Bern, Huber.

Institut fédéral pour la formation et l'enseignement professionnels (BIBB) (2017a), Rangliste 2017 der Ausbildungsberufe nach Anzahl der Neuabschlüsse, BIBB, Bonn, https://www.bibb.de/de/72621.php. Tabelle 67, Accessed 21 November 2018.

Institut fédéral pour la formation et l'enseignement professionnels (BIBB) (2017b), Rangliste 2017 der Ausbildungsberufe nach Neuabschlüssen - Frauen, Bundesinstitut für Berufsbildung, Bonn, https://www.bibb.de/de/72621.php. Tabelle 69, Accessed 21 November 2018.

Institut fédéral pour la formation et l'enseignement professionnels (BIBB) (2017c), Datenblatt 8110 Medizinische/-r Fachangestelltel-r, Bundesinstitut für Berufsbildung, Bonn, https://www2.bibb.de/bibbtools/tools/dazubi/data/Z/B/30/1205.pdf, Accessed 21 November 2018.

Institut fédéral pour la formation et l'enseignement professionnels (BIBB) (2013), Stark besetzte Ausbildungsberufe 2013, Bundesinstitut für Berufsbildung, Bonn, http://www. bibb.de/de/7878.php. Accessed 17 May 2017. 
Institutfédéral pourlaformation etl'enseignement professionnels(BIBB) (2014), Datenblatt 81102820 Medizinische/-r Fachangestelltel-r 2014, Bundesinstitut für Berufsbildung, Bonn. http:/www.bibb.de/bibbtools/tools/dazubi/data/Z/B/30/81102820.pdf. Accessed 27 November 2015.

Institut fédéral pour la formation et l'enseignement professionnels (BIBB) (2007), Medizinische Fachangestellte / Medizinischer Fachangestellter. Erläuterungen und Praxishilfen zur Ausbildungsordnung. Bonn: BW Bildung und Wissen.

Kanning U.P. (2005), Soziale Kompetenzen. Entstehung, Diagnose und Förderung, Göttingen, Hogrefe.

Kanning U.P. (2009), Diagnostik sozialer Kompetenzen. Göttingen, Hogrefe.

Kanning U.P., Schuler H. (2014), „Simulationsorientierte Verfahren der Personalauswahl“, in Schuler H., Kanning P.U. (eds.), Lehrbuch der Personalpsychologie, Göttingen, Hogrefe, pp. 215-256.

Klieme E. (2004), „Was sind Kompetenzen und wie lassen sie sich messen?“, Pädagogik, 6, pp. 10-13.

Langer I., Schulz von Thun F., Tausch R. (1999), Sich verständlich ausdrücken, München, Ernst Reinhardt.

Klieme E., Hartig J. (2007), „Kompetenzkonzepte in den Sozialwissenschaften und im erziehungswissenschaftlichen Diskurs“, Zeitschrift für Erziehungswissenschaft, $\mathrm{n}^{\circ} 10$, Sonderheft 8, Kompetenzdiagnostik, pp. 11-29.

Langer I., Schulz von Thun F., Tausch R. (1999), Sich verständlich ausdrücken, München, Ernst Reinhardt.

Lievens F., Chan D. (2010), "Practical intelligence, emotional intelligence, and social intelligence", in Farr J. L., Tippins N. T. (eds.), Handbook of employee selection, New York, Lawrence Erlbaum, pp. 339-360.

Lorig B., Bretschneider M., Gutschow K., Mpangara M., Weber-Höller R. (2014), Kompetenzorientierte Prüfungen im dualen System - Bestandsaufnahme und Gestaltungsperspektiven, Abschlussbericht, Bonn, Bundesinstitut für Berufsbildung.

Mertens D. (1974), „Schlüsselqualifikationen. Thesen zur Schulung für eine moderne Gesellschaft“, Mitteilungen aus der Arbeitsmarkt- und Berufsforschung 7, n 1, pp. 36-43.

Mischo C. (2004), „Fördert Gruppendiskussion die Perspektiven-Koordination?“, Zeitschrift für Entwicklungspsychologie und Pädagogische Psychologie, n 36, pp. 30-37.

Nickolaus R., Seeber S. (2013), „Berufliche Kompetenzen: Modellierungen und diagnostische Verfahren", in Frey A., Lissmann U., Schwarz B. (eds.), Handbuch Berufspädagogische Diagnostik, Weinheim, Beltz. 
Piaget J. (1973), Das moralische Urteil beim Kinde, Frankfurt A.M., Suhrkamp.

Piaget. J. (2003), Meine Theorie der geistigen Entwicklung. Weinheim, Beltz.

Rosenberg M.B. (2006), Gewaltfreie Kommunikation. Aufrichtig und einfühlsam miteinander sprechen. Neue Wege in der Mediation und im Umgang mit Konflikten, Paderborn, Junfermann.

Ross L. (1977), “The intuitive psychologist and his shortcomings: Distortions in the attribution process", Advances in Experimental Social Psychology, $\mathrm{n}^{\circ} 10$, pp. 173-220.

Roth H. (1971), Pädagogische Anthropologie. Band 2 Entwicklung und Erziehung, Hannover: Herrmann Schroedel.

Seyfried B. (1995), Stolperstein Sozialkompetenz: Was macht es so schwierig, sie zu erfassen, zu fördern und zu beurteilen?, Bielefeld, W. Bertelsmann

Schuler H., Barthelme D. (1995), "Soziale Kompetenz als berufliche Anforderung“, in Seyfried B. (ed.), Stolperstein Sozialkompetenz: Was macht es so schwierig sie zu erfassen, zu fördern und zu beurteilen? Bielefeld, W. Bertelsmann.

Schulz von Thun F. (2004), Klarkommen mit sich selbst und anderen: Kommunikation und soziale Kompetenz - Reden, Aufsätze, Dialoge, Reinbek, Rowohlt.

Selman R. L. (2003), The promotion of social awareness: powerful lessons from the partnership of developmental theory und classroom practice, New York, Russel Sage Foundation.

Tschöpe T., Monnier M. (2016), „Modellierung, Messung und Förderung sozial-kommunikativer Kompetenzen von Medizinischen Fachangestellten in der Ausbildung: Ableitungen aus dem Projekt CoSMed“, Zeitschrift für Berufs-und Wirtschaftspädagogik, Bd. $112, \mathrm{n}^{\circ} 4$, pp. 525-554.

Van Buer J., Matthäus S. (1994), Kommunikative Alltagskultur in der beruflichen Erstausbildung - Ansprüche und Befunde, in Studien zur Wirtschaft- und Erwachsenenpädagogik aus der Humboldt-Universität zu Berlin, pp. 36-120.

Wittmann E. (2001), „Zu kundenkommunikativ kompetentem Handeln und zum Einfluss betrieblicher Ausbildungsbedingungen - Theoretische Überlegungen, empirische Befunde und Anregungen zur praktischen Bedeutsamkeit am Beispiel des Ausbildungsberufs Bankkaufmann/Bankkauffrau“, Zeitschrift für Berufs- und Wirtschaftspädagogik, Beiheft 16, pp. 104-110.

Zapf D. (2002), "Emotion work and psychological well-being: a review of the literature and some conceptual considerations", Human Resourse Managment Review n ${ }^{\circ} 12$, pp. 237-268. 